\title{
Migration of Abdominal Pain - An Effective Tool to Identify Appendicitis and the Only Parameter to Screen
}

\author{
Vikram Panjabrao Vaidya ${ }^{1}$, Yashwant R. Lamture ${ }^{2}$, Harshal Ramteke ${ }^{3}$, Aditya Mundada ${ }^{4}$, Varsha Gajbhiye ${ }^{5}$, \\ Minakshi Yeola6 \\ ${ }^{1}$ Department of Surgery, Jawaharlal Nehru Medical College, Wardha, Maharashtra, India. ${ }^{2}$ Department of \\ Surgery, Datta Meghe Medical College, Nagpur, Maharashtra, India. ${ }^{3}$ Department of Surgery, Jawaharlal \\ Nehru Medical College, Wardha, Maharashtra, India. ${ }^{4}$ Department of Surgery, Jawaharlal Nehru Medical \\ College, Wardha, Maharashtra, India. ${ }^{5}$ Department of Pharmacology, Datta Meghe Medical College, Nagpur, \\ Maharashtra, India. ${ }^{6}$ Department of Surgery, Jawaharlal Nehru Medical College, Wardha, Maharashtra, India.
}

\section{ABSTRACT}

\section{BACKGROUND}

Acute appendicitis is a common disease. It may be tough to identify even with radiological and laboratory investigations. Migratory pain in the abdomen is a helpful key symptom, provided the patient presents within 24 hours of onset of appendicitis.

\section{METHODS}

Patients clinically presenting as acute appendicitis and operated for appendicectomy were recruited in the study. The presence and absence of migratory pain in the abdomen were evaluated and compared with the histopathology of the appendix. Parameters of diagnostic accuracy were measured.

\section{RESULTS}

The sensitivity and specificity of migration of abdominal pain was found to be 97 percent and 100 percent respectively. The positive predictive value and the negative predictive value was 100 percent and 94 percent respectively. Overall diagnostic accuracy was $98 \%$.

\section{CONCLUSIONS}

Migratory pain in the abdomen is a significant symptom to identify as well to rule out acute appendicitis.

\section{KEY WORDS}

Somatic Pain, Blumberg Sign, Appendectomy, Right Iliac Fossa
Corresponding Author: Dr. Yashwant R. Lamture, Professor, Department of Surgery, Datta Meghe Medical College, Nagpur, Maharashtra, India. E-mail:yash18671@gmail.com

DOI: $10.14260 /$ jemds/2020/506

How to Cite This Article:

Vaidya VP, Lamture YR, Ramteke H, et al. Migration of abdominal pain - an effective tool to identify appendicitis and the only parameter to screen. J Evolution Med Dent Sci 2020;9(33):2329-2333, DOI: 10.14260/jemds/2020/506

Submission 24-04-2020,

Peer Review 08-07-2020

Acceptance 13-07-2020,

Published 17-08-2020.

Copyright (C) 2020 JEMDS. This is an open access article distributed under Creative Commons Attribution License [Attribution 4.0 International (CC BY 4.0)] 


\section{BACKGROUND}

Abdominal pain is a key ailment of acute appendicitis (AA). AA is a common emergency in surgery. It has less possibility of death and complications if treated early. Appendicitis was known for more than 100 years and still, it is not without negative laparotomies. Unfortunately, even today, it is associated with a significantly high negative laparotomy rate of $20-25 \%$. Migration of pain is a very important distinguishing interpreter of acute appendicitis. Initially around the umbilicus and then shifting to right iliac fossa suggests the possibility of appendicitis. ${ }^{1}$

Since there present a bunch of disorders of gynaecological, urological, and bowel sites, which have a similar presentation like appendicitis lead to a very difficult situation to diagnose it without error. Moreover, there are patients with atypical presentation adding difficulty in treating clinicians. On the other hand, a physician's ability to find correct diagnosis is variable person to person and its correctness depends on (i) the strength of their basic knowledge, (ii) correctness of history, and last is (iii) accurate interpretation. Deviations from a correct understanding of the AA may cause over or under-diagnosis, it happens even today. Here suggests the need of diagnosis supportive agents that can reinforce correctness and assist to decrease appendicectomy-related complications. Laboratory investigations such as leucocyte count, neutrophilia, and C-reactive protein (CRP) are helpful tools advised in a patient with doubtful patients of acute appendicitis. Supplementary methods such as diagnostic laparoscopy, diagnostic scanning like ultrasonography and computed tomography, clinical scoring systems, and neural networks can be used if available. These tools may assist the surgeon to increase diagnostic accuracy. Styrud, J. et al proved in his study that ultrasound and computed tomography investigations on patients with atypical or doubtful appendicitis are of great significance. Computed tomography is having a higher diagnostic accuracy than ultrasound. In women with reproductive age groups, an unnecessary operation can be avoided and the computed tomography investigation or ultrasound examination are better alternatives to diagnostic laparoscopy. ${ }^{2}$

Acute appendicitis is a common surgical disease with a lifetime incidence of $6 \%$ and most affected somewhat between 11-32 years of age but can occur at any age. A male is more commonly affected than females. The overall lifetime risk is 8.4\% for males and 6.8\% for females in the America. Around 3 lakh appendectomies are done in the United States each year and more than 40,000 cases of acute appendicitis are admitted to the surgery department in the united kingdom per year. While uncomplicated acute appendicitis is having less than $1 \%$ death rate, this increases to $5 \%$ or more for younger children and old patients due to the more possibility of perforation and complications due to late diagnosis. Even today the ideal option for treatment of appendicitis is appendectomy. As early treatment of appendicitis is required to decrease morbidity and deaths, certain relaxations of making overdiagnosis is agreed by most of the surgical schools. An aggressive treatment by the surgeon for doubtful patients of acute appendicitis (i.e., overdiagnosis) will lead to more chances of negative laparotomy; whereas a conservative treatment (i.e., under-diagnosis) will cause a higher rate of perforation and other complications. Hence the treating physician is having a difficult task of making correct equilibrium between overdiagnosis and under diagnosis. Here, diagnosis supportive agents could be of great importance by helping the surgeon to operate and remove appendix early and thereby decreases both negative laparotomy rate and incidence of perforations and deaths. However, these diagnosis supportive agents come with their group of drawbacks and these are discussed below. While the significance of diagnostic laparoscopy has proved to decrease the chances of negative laparotomy by around $25 \%$ and may be useful in doubtful cases, especially in women of reproductive age group or in with high body mass index; but it should be used only after all other methods and investigations, as it is not non-invasive intervention and has associated potential of infection and complications. The significance of diagnostic imaging for doubtful cases of acute appendicitis has improved in modern years. But, Ultrasonography (USG) and Computerized Tomography (CT) cannot be used without the aim for all cases having right-sided abdominal pain because these methods are costly, not available in all hospital and have disadvantage of ionizing radiations (e.g. CT) and operator dependent diagnostic ability (e.g. USG). Few studies suggested that USG and CT scanning in the diagnosis of acute appendicitis, should be done only in those patients who have a difficulty in diagnosis of appendicitis by clinical and laboratory tests. As USG is having a disadvantage of operator dependency and needs experienced person, it is preferable to use CT to USG, as CT has greater diagnostic accuracy. CT scan and USG both are used to assess patients with a doubtful diagnosis of appendicitis. As already stated CT scan has better sensitivity and specificity in comparison to USG with sensitivity and specificity. Still bad part is that both imaging modalities are not able to reduce the negative appendectomy rate. As rural Indian hospitals lack the availability of modern imaging studies and manpower. Patients are usually underdiagnosed or overdiagnosed and based on guesswork, they are referred to higher centers for treatment. This is costly, as patients spend a lot of (i) valuable time to reach higher centers and in waiting for the specialists and (ii) money in traveling and ma imaging studies and manpower. Patients are usually underdiagnosed or over-diagnosed and based on guesswork, they are referred to higher centers for treatment. This is costly, as patients spend a lot of (i) valuable time to reach higher centers and in waiting for the specialists and (ii) money in traveling and maintenance. ${ }^{3}$

Approximately one-third of patients with acute appendicitis manifest pain outside the right lower part of the abdomen. Pain in the left lower quadrant is a particularly rare presentation of acute appendicitis, and most such cases involve congenital abnormalities, including situs inversus totalis and midgut malrotation in which the appendix is located on the left of the abdomen. Hou et al reported a patient with appendicitis who developed pain in the left lower quadrant and had a long, swollen appendix, with the tip pointing toward the presacral region, just across the midline of the lower abdomen. Yang et al reported a patient with acute appendicitis and left lower quadrant pain who had a redundant, floating ascending colon and inflammatory appendix adhering to the descending colon over the left lower abdomen. These cases suggest that the tip of the appendix can be located in many locations beyond the right lower abdominal cavity, due to it causes pain outside of right lower quadrant. The appendix is considered as a mobile organ that 
freely moves inside the abdominal cavity, and its position may not be fixed in a living person. Hence suggests that the location of abdominal pain for recurrent appendicitis can differ from that for the initial episode of appendicitis. Wherever the pain is, physicians need to consider recurrent appendicitis in patients with abdominal pain who had previously undergone conservative treatment of initial appendicitis. Pain in the abdomen due to appendicitis is the most common cause for any age group. Most of the time it is a dilemma to a surgeon to evaluate cases of acute right iliac fossa pain. ${ }^{4}$ Even with the help of various diagnostic scores and investigations like ultrasonography, C-reactive proteins, computed tomography. It is not possible to reduce the rate of removal of normal appendices (15\% to $30 \%) .{ }^{5}$

It has been suggested that the features of abdominal pain and other associated complaints are reliable indicators to identify AA. As abdominal pain is the most common complaint of AA, this study proposes a simple method that takes into account migration of abdominal pain, to reinforce the diagnosis of a patient as a case of AA or no appendicitis (NA). Hence we conducted this study to ascertain the significance of migratory pain in the abdomen in acute appendicitis.

\section{METHODS}

The present study was undertaken in the department of surgery, Datta Meghe Medical College Hingana, Nagpur, in collaboration with Jawaharlal Nehru Medical College, Datta Meghe institute of medical science (DMIMS), Sawangi, Meghe, Wardha, Maharashtra India. This study was a prospective observational. Duration of this study was from January 2019 to January 2020. The numbers of patients were 100 .

\section{Sample Size}

The sample size was calculated as per formula was around 82 Hence 100 sample size was sufficient.

$N=2 \frac{(\mathrm{Z} \alpha+\mathrm{Z} 1-\beta) 2 \sigma 2}{\Delta 2}$

\section{Inclusion Criteria}

Patients between 15-60 years of clinically suspected cases of acute Appendicitis, operated for appendicectomy.

\section{Exclusion Criteria}

1. Pregnancy.

2. Appendicular mass or abscess.

3. Duration of pain in abdomen of more than 48 hours.

4. Urinary calculi and gynaecological diseases.

The data collected included the presenting complaints, clinical signs, laboratory investigations (white cell count more than 10000 and CRP more than $15 \mathrm{mg} / \mathrm{dL}$ ), and ultrasonography. After appendectomy specimen was sent for histopathological examination. The diagnosis of AA was confirmed with histopathology as it is a gold standard investigation.

\section{Statistical Analysis}

The sensitivity, specificity, positive predictive value (PPV), negative predictive value (NPV), and diagnostic accuracy of migratory pain in abdomen derived using SPSS 17.0 statistical software. Ethical approval for the study was obtained from the ethics committee of DMIMS University.

\section{RESULTS}

Around 100 patients included in this prospective study. 60 males and 40 females were present. Age ranges from 19 to 60 years but the majority of patients were in the younger age range of 20 to 30 years (38\%).

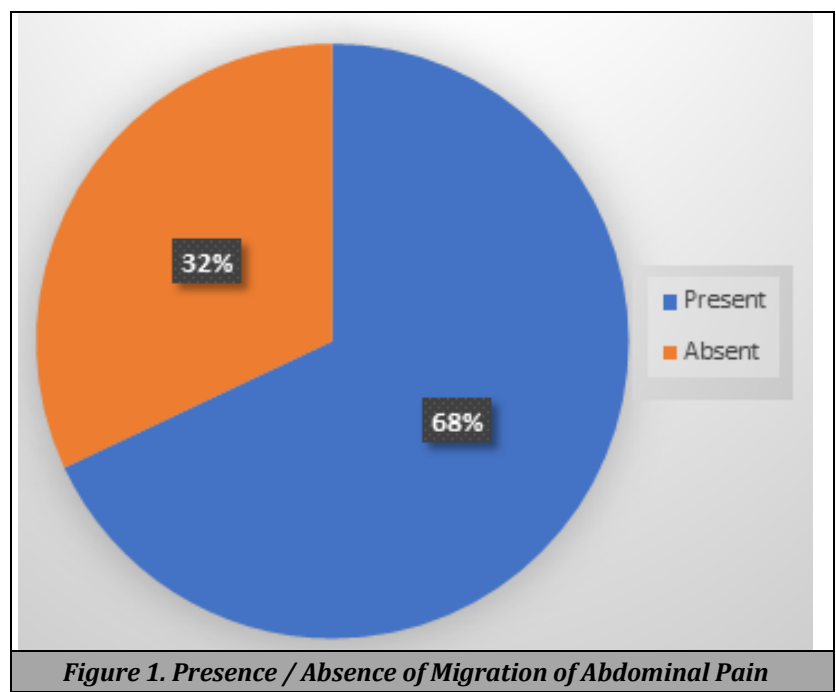

Out of 100 patients 68 patients present with migratory pain in the abdomen. All these patients correctly correlate with acute appendicitis. Remaining 32 patients do not have a history of migration pain but based on clinical judgment with support of investigations undergone appendectomy but found to have a normal appendix in 30 patients and appendicitis in only two patients.

\begin{tabular}{|ccccc|}
\hline Sensitivity Specificity & $\begin{array}{c}\text { Positive } \\
\text { Predictive } \\
\text { Value }\end{array}$ & $\begin{array}{c}\text { Negative } \\
\text { Predictive } \\
\text { Value }\end{array}$ & $\begin{array}{c}\text { Diagnostic } \\
\text { Accuracy }\end{array}$ \\
$97 \%$ & $100 \%$ & $100 \%$ & $94 \%$ & $98 \%$ \\
\hline \multicolumn{2}{|c|}{ Table } & 1. Diagnostic & Accuracy of Migration of Abdominal Pain \\
\hline
\end{tabular}

The sensitivity and specificity of migration of abdominal pain were found to be 97 percent and 100 percent respectively. The positive predictive value and the negative predictive value was 100 percent and 94 percent respectively. The diagnostic accuracy was $98 \%$.

\section{DISCUSSION}

The classical presentation of acute appendicitis starts with diffuse colicky abdominal pain. This is due to mid-gut visceral irritation (appendicular inflammation and obstruction). The pain is initially present in the periumbilical area, but less 
severe than, the colic of the small intestine. Periumbilical abdominal pain is associated with anorexia, nausea, and have one or two episodes of vomiting. Anorexia is a very important clinical feature, particularly in the paediatric age group. The patient often gives a history of similar episodes. Family history is significant as up to one-third of children with appendicitis have a first-degree relative with appendicitis. With continued inflammation of the appendix, the parietal peritoneum in the right iliac fossa becomes inflamed, leading to more severe, constant, and localized somatic pain. Patients often consider it as shifting of abdominal pain. Usually, coughing or sudden movement increases the intensity of pain. ${ }^{6}$

Abdominal pain is the prime feature of acute appendicitis. Centrally located colicky diffuse pain with vomiting then shifting of pain to the right lower quadrant was as per Murphy. This typical sequence is present in $50 \%$ of patients ${ }^{5}$. In the present study, it was in $68 \%$ of the patients (Figure 1).

In a study done by Lamture et al,7 Migratory pain was present in 231 (55.26\%) cases with sensitivity and specificity of $56.67 \%$ and $64.29 \%$ respectively. PPV was $95.67 \%$ and NPV was $9.62 \%$. The results of the present study are much better than these (Table 1).

Both the Yash scoring system and Alvarado scoring system uses migratory abdominal pain as its one important component.7,8 The only limitation of this parameter is that it is not present in all cases of acute appendicitis especially in atypical presentations where it is more somatic or visceral and diffuse. Atypical pain is more common in the old, in whom localization to the right iliac fossa is rare. An inflamed pelvic appendix never produces somatic anterior abdominal wall pain, but may instead cause bladder or rectal symptoms. ${ }^{8}$

Balaji et $\mathrm{al}^{9}$ found to have a pelvic position in around 23 $\%$ cases in his study of 418 cases of acute appendicitis.

So, it is obvious that in these patients, typical shifting of pain may not present.

Classical pain in the abdomen present within 48 hours of duration of its onset is very important. As for more than two days usually associated with perforation or other complications. Hence pain in the abdomen beyond 48 hours is not a reliable predictor of appendicitis ${ }^{1}$. This one is another drawback of this symptom to diagnose acute appendicitis.

Pain in left iliac fossa is usually associated with congenital anomalies like midgut malrotation and situs inversus. Here the appendix is located on the left side of the abdomen. Around $33 \%$ of patients do not have pain in the right lower quadrant of abdomen. ${ }^{5}$ Tetsunori Ikegami et $\mathrm{al}^{4}$ reported a case 27-year-old lady with pain in abdomen initially present in epigastrium, later on, shifted to left iliac fossa. Tenderness was the present left side of the umbilicus with fever and leucocytosis without vomiting and anorexia. Her Alvarado score was suggestive of appendicitis. Computed tomography (CT) reveals a $9 \mathrm{~mm}$ appendix on the left side of the abdomen. Malrotation of the gut with appendicitis was confirmed after surgery, so it is obvious that even in the presence of typical shifting of pain sometimes additional investigations like CT may require.

Regarding migratory pain, the initial pain is completely different in character and origin from the pain which appears in the right iliac fossa after few hours. The initial pain is felt in the center of the abdomen, and though the patient may refer it to the -mid-line at or above the umbilicus, he often describes it as "all across," sweeping his hand evenly across the central region of the abdomen is very vaguely localized. The pain is usually described by the patient as "like an ordinary abdominal pain, but- more severe." It is often griping in nature and variable in severity; the more intense spasms often occur at more or less regular intervals and last for a few seconds. This early pain is entirely not with any tenderness on palpation, and the patient may rub or press on his abdomen without relief. This, initial pain of appendicitis is a visceral splanchnic pain, and is due to increased tension on the muscular wall of the appendix. Some sort of obstruction to the lumen of the appendix, causing accumulation of inflammatory exudate distal to the obstruction, is an essential element in its production. Contrary to common belief the pain disappears after the appendix becomes gangrenous. The short intermittent colicky central abdominal pain so common in children, and described by physicians as "umbilical colic," are very often due to efforts to expel a faecolith or threadworms bundle out of the appendix. Although the visceral pain in acute appendicitis is felt in the umbilical or lower epigastric region, and not in the region of the appendix, it should not be described as a referred or reflected pain, as no radiation of pain or reflex process is involved. The appendix is developmentally a part of the mid-gut loop. The brain can only appreciate painful stimuli arising from any portion of the mid-gut is vaguely situated in the center of the abdomen, and pain of appendicular origin is no exception to the rule. 10

Abdominal pain is the prime feature of acute appendicitis. Centrally located colicky diffuse pain with vomiting then shifting of pain to the right lower quadrant was as per Murphy. This typical sequence is present in $50 \%$ of patients ${ }^{3}$. In the present study, it was in $68 \%$ of the patients.

In a study done by Lamture et al, ${ }^{5}$ Migratory pain was present in 231 (55.26\%) cases with sensitivity and specificity of $56.67 \%$ and $64.29 \%$ respectively. PPV was $95.67 \%$ and NPV was $9.62 \%$. The results of the present study are much better than these.

Both the Yash scoring system and Alvarado scoring system uses migratory abdominal pain as its one important component.5,6 The only limitation of this parameter is that it is not present in all cases of acute appendicitis especially in atypical presentations where it is more somatic or visceral and diffuse. Atypical pain is more common in the old, in whom localization to the right iliac fossa is rare. An inflamed pelvic appendix never produces somatic anterior abdominal wall pain, but may instead cause bladder or rectal symptoms. ${ }^{6}$ Balaji et $\mathrm{al}^{7}$ found to have a pelvic position in around $23 \%$ cases in his study of 418 cases of acute appendicitis. So it is obvious that in these patients, typical shifting of pain may not present.

Classical pain in the abdomen present within 48 hours of duration of its onset is very important. As for the duration of more than two days usually associated with perforation or other complications. Hence pain in the abdomen beyond 48 hours is not a reliable predictor of appendicitis ${ }^{1}$. This one is another drawback of this symptom to diagnose acute appendicitis.

Pain in left iliac fossa is usually associated with congenital anomalies like midgut malrotation and situs inversus. Here the appendix is located in the left side of the abdomen. Around 
$33 \%$ of patients do not have pain in the right lower quadrant of abdomen. ${ }^{3}$ Tetsunori Ikegami et $\mathrm{al}^{2}$ reported a case 27 -yearold lady with pain in abdomen initially present in epigastrium, later on, shifted to left iliac fossa. Tenderness was the present left side of the umbilicus with fever and leucocytosis without vomiting and anorexia. Her Alvarado score was suggestive of appendicitis. Computed tomography (CT) reveals $9 \mathrm{~mm}$ appendix on the left side of the abdomen. Malrotation of the gut with appendicitis was confirmed after surgery, so it is obvious that even in a presence of typical shifting of pain sometimes additional investigations like CT may require.

\section{CONCLUSIONS}

Migratory pain in the abdomen is a significant symptom to identify as well to rule out acute appendicitis which can be seen in half of the patients of acute appendicitis.

Authors would like to acknowledge the Department of Surgery, Datta Meghe Institute of Medical Sciences, Nagpur.

\section{REFERENCES}

[1] Chattopadhyay S, Rabhi F, Acharya UR, et al. An approach to model right iliac fossa pain using pain-only-parameters for screening acute appendicitis. J Med Syst 2012;36(3):1491-502.

[2] Styrud J, Josephson T, Eriksson S. Reducing negative appendectomy: evaluation of ultrasonography and computed tomography in acute appendicitis. Int J Qual Health Care 2000;12(1):65-8.

[3] Schwartz SI. Appendix. In: Schwartz SI, ed. Principles of surgery. $6^{\text {th }}$ edn. New York: McGraw Hill 1994;1307-18.

[4] Ikegami T, Kuriyama A. One appendix, two different pains. Am J Med 2016;129(4):e11-12.

[5] Humes DJ, Simpson J. Acute appendicitis. BMJ 2006;333(7567):530-4.

[6] Mulsow J. The vermiform appendix. Chap- 72. In: Williams N, O'Connell PR, McCaskie AW, eds. Bailey \& Love's short practice of surgery. $27^{\text {th }}$ edn. Boca Raton, FL: CRC Press 2018:1299-317.

[7] Lamture YR, Ramteke H, Shinde RK, et al. Clinico sonological and laboratory co-relation with histopathology of acute appendicitis to develop a new diagnostic scoring system (Yash scoring system). Int Surg J 2017;4(8):2556-64.

[8] Singh CA, Singh AP, Sajith BSM, et al. Role of alvarado score in diagnosis and management of acute appendicitis. Int J Sci Stud 2016;4(6):173-7.

[9] Lamture YR, Salunke B. Anatomical variations related to the position of the appendix. J Evolution Med Dent Sci 2018;7(46):5030-3.

[10] Morley J. Abdominal pain as exemplified in acute appendicitis: a clinical and biological consideration. $\mathrm{Br}$ Med J 1928;1(3516):887-90. 